\author{
Scientific Electronic Archives \\ Issue ID: Sci. Elec. Arch. Vol. 13 (5) \\ May 2021 \\ DOI: http://dx.doi.org/10.36560/14520211350 \\ Article link: https://sea.ufr.edu.br/SEA/article/view/1350
}

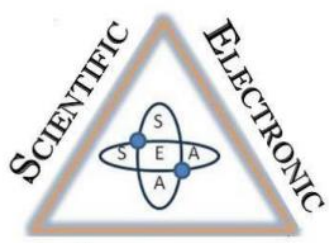

Archives

ISSN 2316-9281

\title{
Feline cryptococcosis attended in the municipality of Sinop: Case report
}

\author{
Corresponding author \\ Caroline Stéphanie Rodrigues Carvalho \\ Federal University of Mato Grosso \\ caroline.src8@gmail.com
}

Kairo Adriano Ribeiro de Carvalho

Federal University of Mato Grosso

Thaís Badini Vieira

Federal University of Mato Grosso

Bruno Gomes de Castro

Federal University of Mato Grosso

\begin{abstract}
Cryptococcosis is a non-contagious systemic mycosis caused by yeasts of the Cryptococcus genus, which can affect humans, domestic and wild animals. Transmission occurs through the inhalation of these airborne yeasts from contaminated environments. In domestic cats, it stands out for infection of the upper respiratory tract, of the cutaneous system, which can reach the central nervous system and still present itself in a disseminated form. Treatment is carried out through the use of antifungals such as fluconazole, itraconazole and amphotericin B for long periods. This article rehearses a case of cryptococcosis in a 10-year-old mixed breed feline with free access to the street. The animal has an ulcerative lesion in the nasal plane, nasal discharge, depression and anorexia. The diagnosis was identified through microbiological culture. Link the severity of the patient's condition to a conduct adopted for euthanasia. Early diagnosis is essential for the establishment of adequate treatment and a favorable prognosis since cryptococcosis, although rare, can be potentially fatal.

Keywords: Mycosis, Cryptococcus, Yeast, Cat.
\end{abstract}

\section{Introduction}

Cryptococcosis is a non-contagious systemic mycosis caused by yeast of Cryptococcus. The Species $C$. neoformans and $C$. gattii are considered more pathogenic and have greater importance in veterinary medicine, having the first one a cosmopolitan distribution (Faria, 2015).

This disease can affect man, domestic and wild animals, having no predisposition to race, sex or age group. Although little observed in the domestic cat clinic, it is the most common systemic ringworm in cats (Grace, 2006).

The growth of Cryptococcus is favored by high concentrations of nitrogen, being commonly associated with substrates such as desiccated pigeon excreta, soil handling locations and hollow trees with decomposing organic matter (Lappin, 2015; Faria, 2015). Inhalation of airborne forms of yeast from contaminated environments is the main source of infection. Therefore, although it can affect men, it is not considered a zoonosis (Penissi et. Al., 2013).

The pathogenesis of cryptococcosis depends on the host's immunocompetence and the virulence of the Cryptococcus strain. The upper respiratory tract is the primary infection site after inhalation of the infectious propagules, causing mycotic rhinitis and destruction of nasal bones. These propagules are small enough to be inhaled into the lower respiratory tract, causing granulomatous lung disease (Penissi et. Al., 2013; Bosco et. Al., 2016).

With the involvement of the nasal cavity, the infection can extend to the central nervous system (CNS) through osteomyelitis of the cribriform plate, in the olfactory bulbs and optic nerves causing meningoencephalitis, optic neuritis and retinitis. In addition, hematogenous and lymphatic dissemination 
may occur causing skin lesions, lymphadenomegaly and involvement of intra-abdominal organs (Greene, 2012; Lappin, 2015).

According to Castellá \& Abarca \& Cabañes (2008), cryptococcosis can be asymptomatic or present in several clinical forms, the nasal form is the most common in cats, in which only the upper respiratory tract is affected leading to sneezing, unilateral or bilateral serous, mucopurulent or hemorrhagic nasal discharge, nodular, ulcerative or polypiform lesions inside and outside the nostrils and swelling of the nasal bridge, a lesion known as "clown nose".

The nasopharyngeal form leads to rales, dyspnea, oral breathing tendency, lymphadenomegaly of the mandibular lymph nodes and ulcerative or proliferative lesions in the oral cavity (Greene, 2012). The pulmonary form is rare, when it occurs, chronic cough and tachypnea are observed (Penissi et. Al., 2013).

In the neurological form, the symptoms vary according to the affected region. However, bizarre behavior, depression, ataxia, seizure and head pressure are the most common signs. Ocular signs can occur in some affected cats and are almost always a marker of CNS involvement, the most common sign of this form are peripheral blindness, mydriasis and unresponsive pupils due to optic neuritis and chorioretinitis. (Trivedi et. Al., 2011).

Skin lesions are common in cats affected by cryptococcosis, usually the result of hematogenous dissemination and are characterized by widespread, painless, floating and firm papules and nodules, with 1 to $25 \mathrm{~mm}$ diameter that may ulcerate and present serous exudate. The localized cutaneous form can rarely develop after direct inoculation of the propagule (Medleau; Hnilica, 2003).

Animals that develop the disseminated form of cryptococcosis have lymphadenomegaly of the thoracic and abdominal lymph nodes, kidney damage and involvement of organs such as spleen and liver. Other reported findings include regional peripheral lymphadenomegaly, bone lysis, arthritis, osteomyelitis, weight loss, apathy and hyporexia (Soltys; Sumner-Smith, 1971; Trivedi et. Al., 2011).

Cryptococcosis can be diagnosed by visualizing yeast in the histopathology of affected tissues and cytology of exudates and tissue aspirates, in addition to serological tests, such as agglutination in latex and ELISA, and mycological culture, allowing the identification of the agent species (Rossi ; Oliveira, 2018).

The most used drugs for cryptococcosis treatment include itraconazole, fluconazole, ketoconazole and amphotericin $B$, and should be used continuously for a long period. The patient in treatment must be followed up until the agent is completely eliminated from the body and the treatment must be extended for at least two months after disappearance of the clinical signs in order to avoid recurrences (Faria, 2015).
Therefore, the objective of this study was to report a case of a feline patient seen at the Veterinary Hospital of the Federal University of Mato Grosso, Sinop campus, diagnosed with cryptococcosis.

\section{Case report}

On July 12, 2019, at the Veterinary Hospital of the Federal University of Mato Grosso - Campus Sinop, a feline, male, unvaccinated, with access to the street, 10 years old and $3.8 \mathrm{~kg}$, presenting a three months ulcerative, rounded and crusted nasal plane lesion reaching the upper lip (Figure 1). In addition to the nasal lesion, the patient also presented an ulcerative lesion with muscle exposure in the left thoracic limb with a history of one year, serous nasal discharge, depression and anorexia. On physical examination, moderate dehydration $(8 \%)$, pale mucous membranes, lean nutritional status, reactive submandibular lymph nodes and pulmonary auscultation were observed. The other parameters were normal.

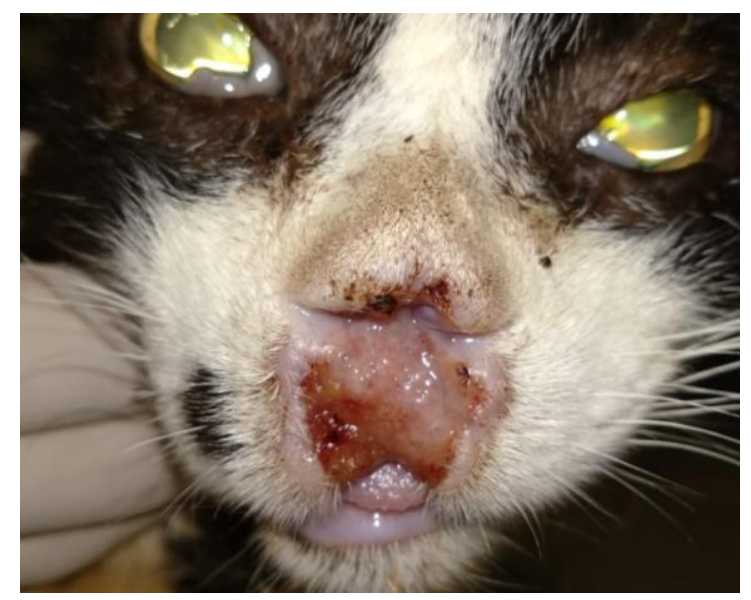

Figure 1: Ulcerative, rounded and crusted lesion in the nasal plane reaching the upper lip. Source: personal archive.

After evaluation of the animal's clinical condition, euthanasia were performed and mycological culture.

For the microbiological diagnosis, samples of the lung, brain and lip of the feline were collected, which were packed separately in sterile petri dishes, duly identified, and immediately sent to the Microbiology Laboratory of the Federal University of Mato Grosso - Campus Sinop (UFMT - Sinop) for microbial isolation.

In the laboratory, the samples were manipulated respecting the safety zone of the Bunsen burner. Before proceeding with the collection of fragments for microbial isolation, sterile scalpel blades were used to perform heat sterilization of the surfaces of the organs, avoiding possible microbial contamination due to the surface of the organ. After this procedure, an incision was made in each organ with a sterile scalpel blade, and a sample of the lung, brain and mouth was collected individually. 
The following protocols were followed for the three organs: fragment of the organ was added to a sterile crucible, followed by the addition of $1 \mathrm{~mL}$ of sterile "Brain Heart Infusion" broth for maceration. After this procedure, $1 \mathrm{~mL}$ of this solution was added to petri dishes containing Sabouraud Agar with chloramphenicol. After ten minutes, the plates were incubated in a bacteriological oven at $35^{\circ} \mathrm{C} \pm 2^{\circ} \mathrm{C}$, being visualized every day until completing one week.

After this period, the same microbial growth was observed in the plates referring to the three organs, characterized by mucoid colonies, shiny, moist and whitish in color (figure 2). The Gram staining technique was then performed, with rounded or oval blue yeast shapes, sometimes with a single monopolar bud, in a light microscope with a 1000x magnification, sometimes showing a clear halo around (figure 3), suggestive of Cryptococcus spp.

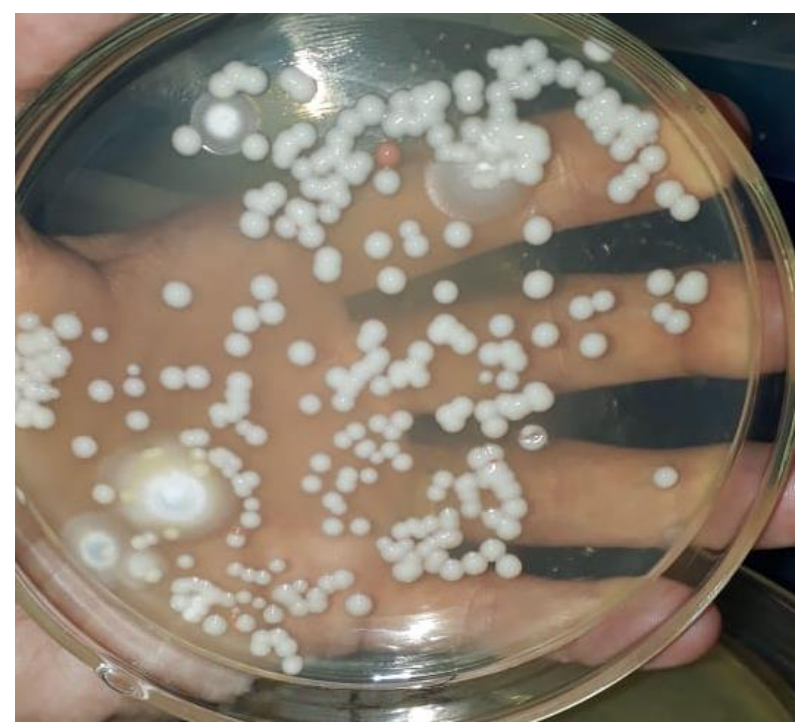

Figure 2: Mucoid colonies, shiny, moist and whitish in color, suggestive of Cryptococcus spp.. Source: Laboratory of Microbiology at UFMT - Sinop ..

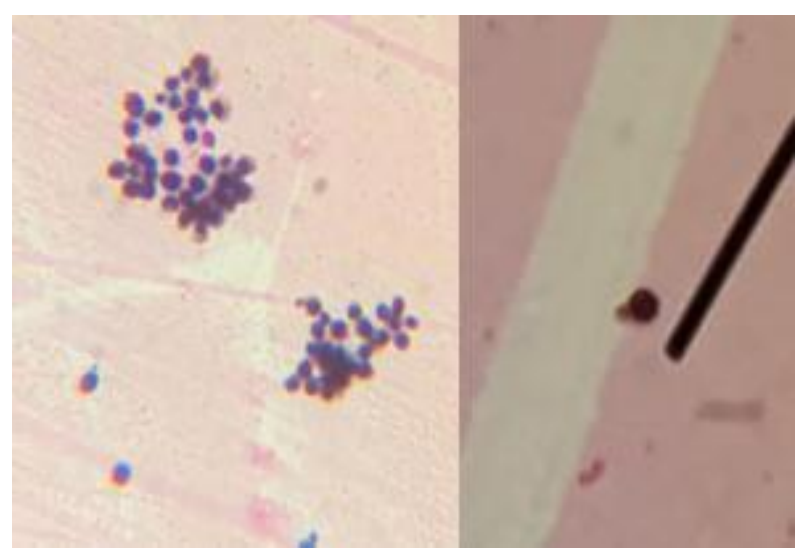

Figure 3: Rounded or oval yeast forms of blue color, sometimes with a single bud, being possible to observe at times a clear halo around. Source: UFMT Microbiology Laboratory - Sinop.

In this case report, the patient studied was a domestic cat, Castellá \& Abarca \& Cabañes (2008) report a greater susceptibility of these animals to infection by Cryptococcus spp. in relation to other animal species. Duncan et. al. (2005) and Trivedi et. al. (2011) also report this greater susceptibility in cats when compared to dogs.

According to Jacobs et. al. (1997), we can associate the gender of the patient in this report with the highest rate of infection by Cryptococcus spp. in males, however, other authors deny the existence of sexual predisposition for this disease, relating the largest casuistry of males with their behavioral characteristics and overrepresentation (O'brien et. al., 2004; Trivedi et.al., 2011).

According to Firacative et. al. (2018) $90 \%$ of human cryptococcosis cases in Latin America are caused by $C$. Neoformans. In the reported patient, the Cryptococcus species was not determined. However, in Brazil, Trilles et. al. (2008) identified the predominance of $C$. Neoformans in the southern macro region and $C$. gattii in the northern macro region, considering human, animal and environmental samples. A study by Daphine et. al. (2014) in the cities of Cuiabá and Sinop pointed out the predominance of $C$. gattii in animal samples and several studies point it as a predominant pathogen in cats. (Trivedi et. Al., 2011; Brito-Santos et. Al., 2019).

The clinical picture of the reported patient started with an increase in volume in the cutaneous region of the left thoracic limb with posterior ulceration and no healing for more than a year. Baes \& Cutsem (1985) and Christianson \& Engber \& Andes (2003) support the possibility of the occurrence of primary cutaneous inoculation of Cryptococcus spp. in humans, however, skin lesions with non-healing may also appear as the first manifestation of the disseminated disease, requiring a complete and systematic diagnostic evaluation to confirm the diagnosis of primary cutaneous cryptococcosis (Neuville et. al., 2003).

The patient's symptoms evolved to nasal cryptococcosis with the presence of disseminated disease and involvement of the lower respiratory tract, skin and lymph nodes. Most studies support the greater occurrence of the nasal form in felines, followed by the nasal form with invasion of nearby sites (such as the central nervous system, retrobulbar space and bridge and nasal plane) and nasal form with dissemination to distant sites (Brito- Santos et. Al., 2019; Trivedi, 2011, O'brien et. Al., 2004).

Due to the high prevalence of nasal shape in cats and its progression, O'brien et. al. (2004) reinforces that, in this species, the primary route of infection by cryptococcosis is the upper respiratory tract and that the CNS is reached from local nasal dissemination through the cribriform plate. Although in humans, the primary route of infection is the lower respiratory tract and dissemination to the CNS is by the hematogenous route (Esher; Zaragoza; Alspaugh, 2018; Griffiths; Kretschmer; Kronstad, 2012).

In the present report, microbiological culture was used as the definitive diagnostic test, considered "gold standard" for cryptococcosis (Malik et. Al., 1996; Pal; Tesfaye; Dave, 2014). However, other tests can 
be used as a screening diagnosis, such as the latex agglutination test (LCAT) and the lateral flow assay for cryptococcal antigen (LFA) (Trivedi et. Al., 2011; Reagan et. Al., 2019 ).

Malik et al. (1996) report that the serological antigen titrations obtained through the LCAT can be used to monitor the patient's response to treatment, as well as the time that must be continued after clinical cure and although it has high specificity (100 $\%)$ and sensitivity (98\%), this test does not rule out the need for confirmatory microbiological culture.

Still in relation to LCAT, Trivedi (2011) correlates a high titer of serum cryptococcal antigen, obtained by this test, with the severity of the disease, contradicting Malik et. al. (1996), who states that this titration does not predict prognosis.

Although presenting lower specificity (84\%) and sensitivity (92\%) for felines when compared to humans, LFA can be used as a screening test for cryptococcosis in this species. Presenting lower cost and greater ease and speed in its performance (Krockenberger et. Al., 2019)

Due to the evolution of the animal's clinical condition and as determined by the Federal Council of Veterinary Medicine: "Euthanasia should be indicated when the treatment represents incompatible costs with the productive activity for which the animal is destined or with the financial resources of the owner ", since the treatment represented costs incompatible with the financial resources of the animal's tutor, was euthanasiaindicated and performed following the guidelines of the Brazilian Guide to Good Practices in Euthanasia in Animals.

If it were possible to opt for treatment, several studies report the efficacy of the drugs amphotericin $\mathrm{B}$, itraconazole and fluconazole (Medleau; Jacobs; Marks, 1995; Malik et. Al., 1992; Malik et. Al., 1996). O'brien et. al. (2006) report that the treatment associated with amphotericin B or with isolated azoles has no significant difference in the final result, however, the average duration of treatment with fluconazole was 4 months and with itraconazole of 9 months showing a significant difference in the duration of treatment. treatment.

Despite the existence of effective treatments, failures continue to occur for several reasons, including resistance of the pathogen to antifungal drugs (Perfect; Cox, 1999). Studies have been developed to create strategies to overcome this obstacle. Brilhante et. al. (2020) report that anthraquinones have a synergistic effect with amphotericin B, its concomitant use enhances the effect of this drug, and may reduce its necessary dosage. Another strategy is the administration of amphotericin B in the liposomal or lipid complex form, which allows the use of higher doses without the toxicity normally found in this drug, especially renal (Coker et. Al., 1993).

Regardless of the choice of the drug used, there are some factors that influence the success of the treatment. Jacobs et. al. (1997) report that the infection site is not related to the final result, however animals with the cutaneous form of the disease are apparently more likely to succeed, corroborating the findings of O'brien et. al. (2006), in which animals that did not respond to treatment tended to have the severe form of cryptococcosis.

In addition, O'brien et. al. (2006) argue that the concomitant presence of Feline Immunodeficiency (IVF) does not have a significant effect on the effectiveness of the treatment, contrasting Jacobs et. al. (1997) who associate this disease with a less favorable prognosis.

Cryptococcosis has no zoonotic potential, it is not transmitted directly from the sick animal to humans, much less between animals of the same species (Castellá; Abarca; Cabañes, 2008). Although, Nosanchuk et. al. (2000) report a strong suggestibility of zoonotic transmission in a case in North America.

Due to the common aggregation of birds in urban centers, environmental exposure, whether human or animal, to infectious sources is difficult to control and constitutes the biggest problem of cryptococcosis in relation to public health. It is recommended to clean these areas regularly, as well as bird breeding sites (Bosco et. Al., 2016).

\section{Conclusions}

Cryptococcosis is a rare systemic mycosis, however potentially fatal, and should be considered as a differential diagnosis in animals presenting skin lesions, symptoms related to the upper respiratory tract and central nervous system. Early diagnosis is important for the institution of adequate treatment.

The control and cleaning of urban areas with the aggregation of birds, as well as breeding grounds, is fundamental for the prevention of this condition.

\section{References}

BAES, H., CUTSEM, JV Primary cutaneous cryptococcosis. Dermatologica 171: 357-361, 1985. https://doi.org/10.1159/000249452.

BOSCO, SMG; LAZÉRA, MS; PACHECO, TMV; BAGAGLI, E. Cryptococcosis. In: MEGID, J .; RIBEIRO, MG; PAES, AC Infectious Diseases in Farm and Companion Animals. 1. ed .: Roca, 2016. ch. 82, p. 878-885. ISBN 9788527727891. BRILHANTE

, R .; ARAÚJO, G .; FONSECA, X. et. al. Antifungal effect of anthraquinones against Cryptococcus neoformans: detection of synergism with amphotericin B. Medical mycology, 2020. myaa081. Advance online publication. https://doi.org/10.1093/mmy/myaa081.

BRITO-SANTOS, F. et al. Cryptococcosis due to Cryptococcus gattii VGII in southeast Brazil: the one health approach revealing a possible role for domestic cats. Medical Mycology Case Reports, 24: 61-64, 2019. doi: 10.1016 / j.mmcr.2019.04.004.

CASTELlÁ, G ; ABARCA, ML; CABAÑES, FJ Criptococosis y 'Animales de Compañía. Rev 
Iberoamericana de Mycologia, Bilbao (Spain), 25: 10191024, 2008. doi: 10.1016 / S1130-1406 (08) 70021-4.

CHRISTIANSON, J. C ;, ENGBER, W .; ANDES, D. Primary cutaneous cryptococcosis in immunocompetent and immunocompromised hosts. Med Mycol., 41 (3): 177-188, 2003. doi: 10.1080 / 1369378031000137224.

COKER, RJ; VIVIANI, M .; GAZZARD, BG et. al. Treatment of cryptococcosis with liposomal amphotericin $B$ (AmBisome) in 23 patients with AIDS. AIDS (London, England). 7 (6): 829-835, 1993. https://doi.org/10.1097/00002030-199306000-00011.

FEDERAL COUNCIL OF VETERINARY MEDICINE CFMV. Brazilian guide to good practices for euthanasia in animals - Recommended concepts and procedures. Brasília, 2013,

http://www.agricultura.gov.br/arq_editor/file/

Desenvolvimento_Sustentavel/Producao-

IntegradaPecuaria/Guia\%20de\%20Boas\%20Pr\%C3\%A1ti cas\%20para\%20Eutanasia_pdf.pdf.

DAPHINE, P. et al. Occurrence and molecular characterization of cryptococcosis in dogs and cats in Mato Grosso, Brazil. Brazilian Veterinary Research. 34: 167-172, 2014. doi: 10.1590 / S0100-736X2014000200012.

DUNCAN, C .; STEPHEN, C .; LESTER, S .; BARTLETT, $\mathrm{KH}$ Sub-clinical infection and asymptomatic carriage of Cryptococcus gattii in dogs and cats during an outbreak of cryptococcosis, Medical Mycology, Volume 43, Issue 6, September 2005, Pages 511-516, https://doi.org/10.1080/13693780500036019

ESHER, S ; ZARAGOZA , O ; ALSPAUGH, J .. Cryptococcal pathogenic mechanisms: A dangerous trip from the environment to the brain. Memories of the Oswaldo Cruz Institute. Rio de Janeiro. 2018. Vol. 113. doi: 10.1590 / 0074-02760180057.

FARIA, RO Infectious Diseases: Dimorphic Fungi and Related to Deep Mycoses.In: JERICÓ, MM; NETO, JPA; KOGIKA, MM Treatise on Internal Medicine for Dogs and Cats. 1. ed. Rio de Janeiro: Roca, 2015. v. 1, ch. 87, p. 784787. ISBN 978-85-277-2643-6.

FIRACATIVE, C .; LIZARAZO, J .; ILLNAIT-ZARAGOZÍ, MT; CASTAÑEDA, E. Latin American Cryptococcal Study Group. The status of cryptococcosis in Latin America. Mem Inst Oswaldo Cruz. 2018; 113 (7): e170554. doi: 10.1590 / 0074-02760170554. Epub 2018 Apr 5. PMID: 29641639; PMCID: PMC5888000.

GRACE, SF Cryptococcosis. In: NORSWORTHY, GD; CRYSTAL, MA; GRACE, SF; TILLEY, LP The Feline Patient. 3. ed. lowa: Blackwell Publishing, 2006. ch. 26, p. 55-58. ISBN 978-0-7817-6268-7.

GREENE, CE Fungal and Algal Diseases: Cryptococcosis. In: GREENE, CE Infectious Diseases of the Dog and Cat. 4. ed. [S. I.]: Elsevier / Saunders, 2012. ch. 59, p. 620-625. ISBN 1416061304.

GRIFFITHS, EJ; KRETSCHMER, M .; KRONSTAD, JW Aimless mutants of Cryptococcus neoformans: failure to disseminate. Fungal biology reviews, 2012. 26 (2-3), p. 6172. https://doi.org/10.1016/j.fbr.2012.02.004.
JACOBS, GJ; MEDLEAU, L .; CALVERT, C .; BROWN, J. Cryptococcal infection in cats: factors influencing treatment outcome, and results of sequential serum antigen titers in 35 cats. Journal of veterinary internal medicine vol. 11.1. 1997: 1-4. doi: 10.1111 / j.1939-1676.1997.tb00064.x.

KROCKENBERGER, M .; MARSCHNER, C .; MARTIN, P. et. al .. Comparing immunochromatography with latex antigen agglutination testing for the diagnosis of cryptococcosis in cats, dogs and koalas. Medical mycology. 2019. p. 1-8. doi: .1093 / mmy / myz010.

LAPPIN, MR Infectious Diseases: Multisystemic Mycotic Infections. In: NELSON, RW; COUTO, CG Internal Medicine for Small Animals. 5. ed. Rio de Janeiro: Elsevier, 2015. cap. 95, p. 1360-1363. ISBN 978-85-352-7906-1.

MALIK, R .; CRAIG, AJ; WIGNEY, DI; MARTIN, P .; LOVE, DN Combination chemotherapy of canine and feline cryptococcosis using subcutaneously administered amphotericin B. Australian veterinary journal, 1996. 73 (4), p. 124-128. https://doi.org/10.1111/j.17510813.1996.tb10003.x.

MALIK, R .; MCPETRIE, R .; WIGNEY, DI; CRAIG, AJ; LOVE, DN A latex cryptococcal antigen agglutination test for diagnosis and monitoring of therapy for cryptococcosis. Australian veterinary journal, 1996. 74 (5), p. 358-364. https://doi.org/10.1111/j.1751-0813.1996.tb15445.x.

MALIK, R .; WIGNEY, DI; MUIR, DB; GREGORY, DJ; LOVE, DN Cryptococcosis in cats: clinical and mycological assessment of 29 cases and evaluation of treatment using orally administered fluconazole. Journal of medical and veterinary mycology: bi-monthly publication of the International Society for Human and Animal Mycology, 1992. $30 \quad$ (2), $\quad$ p. $133-144$. https://doi.org/10.1080/02681219280000181.

MEDLEAU, L .; HNILICA, KA Cutaneous Mycoses: Cryptococcosis. In: MEDLEAU, L ; HNILICA, KA Small Animal Dermatology: Colored Atlas and Therapeutic Guide. 1. ed. São Paulo: Roca, 2003. chap. 3, p. 55-56. ISBN 857241-416-9.

MEDLEAU, L .; JACOBS, GJ; MARKS, MA Itraconazole for the treatment of cryptococcosis in cats. Journal of veterinary internal medicine, 1995. 9 (1), p. 39-42. https://doi.org/10.1111/j.1939-1676.1995.tb03270.x.

NEUVILLE, S .; DROMER, F .; MORIN, O. et al. Primary cutaneous cryptococcosis: a distinct clinical entity. Clin Infect Dis. 2003; 36 (3): 337-347. doi: 10.1086 / 345956.

NOSANCHUK, JD; SHOHAM, S ; FRIES, BC et. al. Evidence of zoonotic transmission of Cryptococcus neoformans from a pet cockatoo to an immunocompromised patient. Annals of internal medicine, 2000. 132 (3), p. 205-208. https://doi.org/10.7326/00034819-132-3-200002010-00006.

O'BRIEN, CR; KROCKENBERGER, MB; MARTIN, P .; WIGNEY, DI; MALIK, R. Long-term outcome of therapy for 59 cats and 11 dogs with cryptococcosis. Australian veterinary journal, 2006. 84 (11), p. 384-392. https://doi.org/10.1111/j.1751-0813.2006.00040.x.

O'BRIEN, CR, KROCKENBERGER, MB, WIGNEY, DI, MARTIN, P., MALIK, R. Retrospective study of feline and 
canine cryptococcosis in Australia from 1981 to 2001: 195 cases. Med Mycol. 2004; 42 (5): 449-460. doi: $10.1080 /$ 13693780310001624547

PAL, M .; TESFAYE, S .; DAVE, P. Cryptococcosis: An Enigmatic Mycosis of Humans and Animals. Journal of Environmental and Occupational Science. 2014. 3. p. 5360. 10.5455 / jeos.20131107122611.

PENNISI, MG; HARTMANN, K ; LLORET, A. et al. CRYPTOCOCCOSIS IN CATS: ABCD guidelines on prevention and management. Journal of Fel. Med. Surg. year 2013, p. 611-618, 15 jul. 2013. doi: 10.1177/ 1098612X13489224. PMID: 23813826.

PERFECT, JR; COX, GM Drug resistance in Cryptococcus neoformans. Drug resistance updates: reviews and commentaries. Antimicrobial and Anticancer Chemotherapy, 1999. 2 (4), p. 259-269. https://doi.org/10.1054/drup.1999.0090.

REAGAN, KL; MCHARDY, I .; THOMPSON, GR 3rd : SYKES, JE Evaluation of the clinical performance of 2 point-of-care cryptococcal antigen tests in dogs and cats. Journal of veterinary internal medicine, 2019. 33 (5), p. 2082-2089. https://doi.org/10.1111/jvim.15599.

ROSSI, CN; OLIVEIRA, CD Main Infectious Cat Diseases: Cryptococcosis in Cats. In: SOLTYS, MA; SUMNERSMITH, G. Systemic mycoses in dogs and cats. Can Vet J. 1971 Oct; 12 (10): 191-9. PMID: 5170146; PMCID: PMC1695444.

TINUCCI-COSTA, M ; DAGNONE, AS. Infectious Diseases in the Routine of Dogs and Cats in Brazil. 1. ed. Curitiba: Medvep, 2018. p. 279-282. ISBN 978-85-6675906-8.

TRILLES, L .; LAZÉRA, MS; WANKE, B. et al. Regional pattern of the molecular types of Cryptococcus neoformans and Cryptococcus gattii in Brazil. Mem. Inst. Oswaldo Cruz, Rio de Janeiro, Vol. 103, n. 5, p. 455-462, Aug. 2008. doi.org/10.1590/S0074-02762008000500008.

TRIVEDI, SR; MALIK, R .; MEYER, W .; SYKES, JE Feline cryptococcosis: impact of current research on clinical management. Journal of feline medicine and surgery, 2011, p. 163-172. https://doi.org/10.1016/j.jfms.2011.01.009. doi: 10.1016 / j.jfms.2011.01.009.

TRIVEDI, SR; SYKES, JE; CANNON, MS et al. Clinical features and epidemiology of cryptococcosis in cats and dogs in California: 93 cases (1988-2010). J Am Vet Med Assoc. 2011; 239 (3): 357-369. doi: 10.2460 / javma.239.3.357. 